\title{
Cyclocarya paliurus (Batal.) Ijinskaja Aqueous Extract (CPAE) Ameliorates Obesity by Improving Insulin Signaling in the Hypothalamus of a Metabolic Syndrome Rat Model
}

\author{
Guangyuan Xu, ${ }^{1}$ Hisae Yoshitomi, ${ }^{2}$ Wen Sun, ${ }^{1}$ Xuan Guo, ${ }^{1}$ Lili Wu, ${ }^{1}$ Xiangyu Guo, ${ }^{3}$ \\ Lingling Qin, ${ }^{1}$ Yixin Fan, ${ }^{1}$ Tunhai Xu, ${ }^{4}$ Tonghua Liu, ${ }^{1}$ and Ming Gao ${ }^{2}$ \\ ${ }^{1}$ Key Laboratory of Health Cultivation of the Ministry of Education, Beijing University of Chinese Medicine, Beijing 100029, China \\ ${ }^{2}$ School of Pharmaceutical Sciences, Mukogawa Women's University, 11-68 Koshien Kyuban-cho, Nishinomiya, Hyogo 663-8179, Japan \\ ${ }^{3}$ Department of Endocrinology, Dongfang Hospital, Beijing University of Chinese Medicine, Beijing 100078, China \\ ${ }^{4}$ School of Chinese Pharmacy, Beijing University of Chinese Medicine, Beijing 100029, China \\ Correspondence should be addressed to Tonghua Liu; thliu@vip.163.com and Ming Gao; gaoming@mukogawa-u.ac.jp
}

Received 8 February 2017; Revised 28 April 2017; Accepted 15 May 2017; Published 8 June 2017

Academic Editor: Roberto K. N. Cuman

Copyright (C) 2017 Guangyuan Xu et al. This is an open access article distributed under the Creative Commons Attribution License, which permits unrestricted use, distribution, and reproduction in any medium, provided the original work is properly cited.

\begin{abstract}
Background. Antiobesity drugs may not be optimal for treating obesity. However novel antiobesity agents, especially those derived from natural products, may be suitable. Therefore, we investigated the effects and mechanisms of Cyclocarya paliurus (CP) aqueous extract (CPAE) on obesity. Methods. SHR.Cg-Leprcp/NDmcr (SHR/cp) rats were used as a model of obesity and metabolic syndrome. Experimental animals were allocated into two groups-control and CPAE $(0.5 \mathrm{~g} / \mathrm{kg})$ - for a 7 -week treatment period. Examinations were performed, including general physiological characteristics, obesity-related biochemical parameters, and insulinsignaling pathway-related proteins in the hypothalamus. Results. Treatment with CPAE reduced food intake, body weight, organ weight, fat mass, and body mass index (BMI) in SHR/cp rats. Meanwhile, CPAE also decreased the levels of fasting serum glucose, fasting serum insulin, HOMA-IR, serum free fatty acids, serum malondialdehyde, serum superoxide dismutase, and serum total-glutathione. The levels of phosphorylation of target proteins-including InsR, IRS1, PI3Kp85, Akt, and FoXO1 as well as protein expression of POMC-were significantly upregulated in the hypothalamus, but NPY expression remarkably decreased. Conclusions. CPAE has antiobesity, antihypoglycemic, antihypolipidemic, and antioxidant properties. The mechanism responsible for the antiobesity effect of CPAE may be related to suppression of energy intake via regulation of insulin-signaling pathway in the hypothalamus.
\end{abstract}

\section{Introduction}

Obesity is a major public health problem approaching epidemic proportions worldwide. It is associated with metabolic comorbidities such as type 2 diabetes mellitus, cardiovascular injury, hyperlipemia, and other obesity-related diseases $[1$, 2]. Therefore, a better understanding of the mechanisms of obesity is urgently needed along with the development of new antiobesity drugs.

The imbalance between energy intake and energy expenditure is the key cause of obesity $[3,4]$. Previous studies revealed that the hypothalamus is a key brain region in the regulation of food intake, and adipose tissue is crucial in energy storage and expenditure. Both play significant roles in maintaining energy homeostasis and in treating obesity [57].

In the hypothalamus, there are two types of appetiteregulating neurons: (a) coexpression of neuropeptide $\mathrm{Y}$ (NPY) and agouti-related protein (AgRP), both of which express melanocortin receptors (MCR) antagonists, and (b) proopiomelanocortin (POMC) which expresses MCR agonists $[8,9]$. In the hypothalamus, POMC and NPY neurons are regulated by different hormones (e.g., leptin and insulin). These hormones make the melanocortin system sensitive to 
changes in both food intake and body weight [10]. Since insulin receptors (InsR) are widely expressed in the hypothalamus, the insulin-signaling pathway plays a key role in the control of feeding activity [11]. Two studies found that diabetes and stress are involved in the dysregulation of insulinsignaling pathway in the hypothalamus [12, 13]. Another study showed that intracerebroventricular (i.c.v.) injection of insulin can inhibit food intake through the regulation of PI3K signaling in the hypothalamus [14]. Moreover, in the hypothalamus, the forehead box-containing protein of the $\mathrm{O}$ subfamily (FoxO)1-a downstream transcription factor of the insulin-signaling pathway-has a dual function: inhibiting the expression of POMC and stimulating the expression of NPY leading to an increased food intake [15, 16]. On the other hand, FoxO1 activities are also regulated by the InsR/PI3K/Akt signaling pathway, in which insulin induces Akt phosphorylation, followed by inactivation of FoxO1 due to its phosphorylation by p-Akt [17].

Inactivation of FoxO1 causes upregulation of POMC expression and downregulation of NPY expression, leading to loss of appetite and weight [18]. On the other hand, insulin resistance in the hypothalamus reduces insulin-mediated Akt phosphorylation, thereby upregulating overexpression of FoxO1, which results in increased food intake and obesity $[19,20]$. Therefore, to control food intake in obesity, targeting the insulin-signaling pathway may be a suitable approach.

Therapeutic intervention via weight-loss drugs (accompanied by diet and exercise) is one option for the treatment and management of obesity. However, antiobesity drugs (e.g., orlistat) currently available or under development in clinical trials may not be optimal for treating morbid obesity with metabolic complications. Thus it may be beneficial to develop novel antiobesity agents, especially those derived from natural products, which often have fewer adverse effects.

Cyclocarya paliurus (CP) (Batal.) Iljinsk (family Cyclocaryaceae) is a traditional medicinal herb, which only grows in southern China [21, 22]. The leaves of CP (commonly known as "sweet tea tree") have been widely used in traditional medicine for the treatment of hypertension, obesity, and diabetes. In addition, CP hypoglycemic tea is the first Chinese health tea approved by the United States Food and Drug Administration (FDA) [23, 24].

$\mathrm{CP}$ aqueous extract (CPAE), one of the main active components of $\mathrm{CP}$, contains many polysaccharide components. Several studies showed that CPAE can improve insulin resistance by inhibiting serine phosphorylation of IRS-1, thereby restoring tyrosine phosphorylation of IRS-1 and increasing Akt phosphorylation in the muscle tissue of mice with adipose dysfunction and insulin resistance [25]. In addition, animal studies showed that CPAE can reduce food intake in high-fat-diet (HFD) and streptozotocin- (STZ-) induced type 2 diabetic rats and can lower lipid levels in hyperlipidemic mice $[26,27]$. However, it is not clear whether CPAE can affect insulin signaling in the hypothalamus. Moreover, the mechanisms underlying the antiobesity effects of CPAE are also rarely reported. Therefore, by identifying the responsible mechanism and possible effect of CPAE on food intake, we can elucidate our knowledge of obesity treatment using CPAE and measure its efficacy.
In this study, we investigated the mechanism and effect of CPAE on obesity using leptin receptor-deficient SHR.CgLeprcp/NDmcr (SHR/cp) rats as a model of morbid obesity with metabolic syndrome (MetS) [28]. Analysis of our results would then reveal the effect of CPAE on the insulin-signaling pathway in the hypothalamus of the leptin receptor-deficient rats.

\section{Materials and Methods}

2.1. Preparation of CPAE. CP leaves were collected from Xiushui County, Jiangxi Provence, and verified at the School of Chinese Materia Medica of Beijing University of Chinese Medicine (Beijing, China) by Professor Tun-hai Xu. The airdried and powdered leaves $(40 \mathrm{~kg})$ were extracted 3 times ( 2 hours/each) with 8 times the amount of $85 \%$ ethanolheating reflux. The extracts were combined, concentrated, and finally dissolved in $40 \mathrm{~L}$ water, followed by a degreasing treatment (flushed 3 times with petroleum ether at a $1: 1$ volume ratio). The water extracts were then freeze-dried after decompression and concentration, leaving the CPAE powder $(600 \mathrm{~g})[29,30]$. The proportion of total polysaccharides in the CPAE powder was approximately $68.11 \%$, as determined by UV-spectrometer.

2.2. Animal Experiment. Male 7-week-old SHR/cp rats (190-210 g) and age-matched male Wistar Kyoto (WKY) rats (150-170 g) were purchased from Japan SLC, Inc. (Shizuoka, Japan). The animals were kept in a specific-pathogen-free (SPF) animal facility at Mukogawa Women's University (Nishinomiya, Japan), given ad libitum access to normal chow and water, and maintained in controlled temperature $\left(22-24^{\circ} \mathrm{C}\right)$, relative humidity $(40-60 \%)$, and $12 / 12 \mathrm{~h}$ light/dark cycle conditions. The care and treatment of the experimental animals were conducted in accordance with the Guidelines for the Care and Use of Laboratory Animals of Mukogawa Women's University. The experimental protocols were approved by the Animal Care and Use Committee of Mukogawa Women's University.

$\mathrm{SHR} / \mathrm{cp}$ rats were a proven model of prediabetes and MetS characterized by hyperglycemia, hyperlipidemia, hypertension, and obesity [26]. The SHR/cp rats were chosen (see Table 1) and randomly divided into two groups, each containing 6 rats: (1) an untreated control group (control group) and (2) rats treated with $0.5 \mathrm{~g} / \mathrm{kg} \mathrm{CPAE} \mathrm{(CPAE} \mathrm{group).} \mathrm{Levels}$ of fasting blood glucose (FBG), total triglycerides (TG), total cholesterol (TC), and free fatty acids (FFA) in the serum, as well as animal body weight, were measured in all rats. CPAE was prepared in sterile water and administered orally once per day for 7 consecutive weeks. Control group rats received the same volume of sterile water.

2.3. General Condition, Organ Weight, and Body Mass Index (BMI) of Experimental Rats. The general condition of the experimental rats was daily monitored, including mental state, body weight, body weight gain, food intake, and survival. Body weight and food intake were recorded every day throughout the experiments. Organs and fat of experimental rats were weighed at the end of treatment. BMI was 
TABLE 1: SHR-cp rats used in the experiments.

\begin{tabular}{lccccccc}
\hline Group & Dose $(\mathrm{g} / \mathrm{kg})$ & FBG $(\mathrm{mg} / \mathrm{dL})$ & Body weight $(\mathrm{g})$ & $\mathrm{TG}(\mathrm{mg} / \mathrm{mL})$ & $\mathrm{CHO}(\mathrm{mg} / \mathrm{dL})$ & FFA $(\mathrm{mEq} / \mathrm{L})$ & SBP $(\mathrm{mmHg})$ \\
\hline Control & - & $94.57 \pm 10.23$ & $172.17 \pm 8.50$ & $274.13 \pm 68.08$ & $64.60 \pm 7.15$ & $1.96 \pm 0.16$ & $130.78 \pm 10.57$ \\
CPAE & 0.5 & $92.10 \pm 9.50$ & $171.50 \pm 5.09$ & $275.66 \pm 48.87$ & $64.08 \pm 6.91$ & $1.96 \pm 0.28$ & $131.28 \pm 5.37$ \\
\hline
\end{tabular}

Data are expressed as the mean \pm SD.

$P<0.05$ and ${ }^{* *} P<0.001$ versus control. Control, control group $(n=6)$; CPAE, Cyclocarya paliurus leaf aqueous extract group $(n=6)$; SHR/cp, SHR.CgLeprcp/NDmcr rat. FBG, fasting blood glucose; TG, total triglycerides; $\mathrm{CHO}$, total cholesterol; FFA, free fatty acids; SBP, systolic blood pressure.

measured by bioelectrical impedance analysis (BIA) (ImpediVet, ImpediMed Ltd., Brisbane, Australia). Acquired data were processed using bioimpedance software (ImpediVet Vet BIS1 v1.0.2).

2.4. Biochemical Analysis. TG, TC, FFA, glutamic pyruvic transaminase (GPT), and glutamic oxaloacetic transaminase (GOT) were measured using commercially available kits (Wako Pure Chemical Industries, Osaka, Japan). Serum creatinine (Screa), malondialdehyde (MDA), superoxide dismutase (SOD), and total-glutathione (T-GSH) were determined by colorimetric assays (SINO-UK Institute of Biological Technology, Beijing, China). The level of serum insulin was analyzed using enzyme-linked immunosorbent assay (ELISA) kits (SINO-UK Institute of Biological Technology, Beijing, China). Quantification of insulin resistance and betacell function was performed using the homeostasis model of assessment-insulin resistance (HOMA-IR) index calculated from the equation: HOMA-IR $=$ FPG $\times$ Fins/22.5 [31].

2.5. Histological Examination of Adipose Tissue. Abdominal adipose tissues were isolated at the end of treatment and fixed in $4 \%$ paraformaldehyde. Tissue samples were embedded in paraffin, sectioned ( $4 \mu \mathrm{m}$ thick), stained with hematoxylineosin (H\&E), and examined under an optical microscope (Olympus BX53, Olympus, Tokyo, Japan).

2.6. Western Blot Analysis. Hypothalamus and adipose tissue samples were collected at the end of treatment and stored at $-80^{\circ} \mathrm{C}$ prior to use. Tissue samples were prepared in a homogenization buffer containing $50 \mathrm{mM}$ Tris- $\mathrm{HCl}(\mathrm{pH}$ 7.4), $100 \mathrm{mM} \mathrm{NaCl}, 1 \%$ Nonidet-P40, $0.25 \%$ sodium deoxycholate, $0.1 \%$ SDS, $1 \mathrm{mM}$ EDTA, $50 \mathrm{mM} \mathrm{NaF}, 2 \mathrm{mM} \mathrm{Na}_{3} \mathrm{VO}_{4}$, $30 \mathrm{mM}$ sodium pyrophosphate, $2 \mathrm{mM}$ PMSF, $1 \mathrm{mM}$ benzamidine, $0.02 \mathrm{~g} / \mathrm{mL}$ trypsin inhibitor, $0.02 \mathrm{~g} / \mathrm{mL}$ leupeptin, and $0.02 \mathrm{~g} / \mathrm{mL}$ aprotinin. Lysates were incubated on ice for $30 \mathrm{~min}$, followed by centrifugation at $15,000 \mathrm{rpm}$ for $30 \mathrm{~min}$ to collect the supernatant. After measurement of protein concentration, supernatants with equal amount of protein were mixed with a loading buffer containing $0.5 \mathrm{mmol} / \mathrm{L}$ Tris/HCl, pH 6.8, glycerol, $10 \%$ SDS, $0.1 \%$ bromophenol blue, and 2-mercaptethanol and then denatured in a boiling water bath.

The protein samples were separated by $7.5 \%-12.5 \%$ SDSPAGE gel electrophoresis, transferred onto a PVDF membrane (Amersham Life Science Inc., Little Chalfont, Buckinghamshire, UK). After being blocked using either Blocking One or Blocking One-p buffer (Nacalai Tesque, Kyoto, Japan), the membranes were incubated at $4^{\circ} \mathrm{C}$ overnight with the primary antibodies, including p-InsR (cat\#, 3024s),
PI3Kp85 (4257s), p-Akt (4060s), p-FoXO1(9461s), and $\beta$ actin antibodies (4970s) (Cell Signaling Technology, USA), as well as POMC (ab94446), NPY (ab180809) (Abcam, Eugene, OR, USA), and p-IRS1tyr989 (L1912) (Santa Cruz Biotechnology Inc., Dallas, TX, USA). The next day, membranes were washed with TTBS and incubated for 1 hour with HRP conjugated anti-rabbit, anti-goat, or anti-mouse IgG antibodies (1:10,000 dilution). The bound antibodies were visualized using enhanced chemiluminescence. The relative levels of interesting proteins to $\beta$-actin (internal control) were analyzed by Image J v7.0 software.

2.7. Statistical Analysis. Data are expressed as mean \pm standard deviation (SD). The difference between two groups was analyzed by Student's $t$-test. For multiple comparisons, the significance of differences among groups was determined by one-way analysis of variance with Bonferroni's correction. $P<0.05$ was considered statistically significant.

\section{Results}

3.1. Effects of CPAE on Obesity and MetS in SHR/cp Rats. As shown in Figure 1, after 1 week of treatment, food intake in CPAE treatment group was significantly lower than that in control group (Figure 1(a)). Therefore, body weight gain in the CPAE group was smaller than that in control group after 1 week of treatment (Figure 1(b)). Moreover, an increase in body weight was found in both groups, but the amount of increased body weight in CPAE group was significantly smaller than that in control group after 2 weeks' treatment (Figure 1(c)). Comparisons of the organ weight differences between the two groups showed that the liver and brain weights in the CPAE group were significantly lower than in the CPAE group after 7 weeks' intervention (Figure 1(d)). BMI in the CPAE group was also significantly lower than in control group rats (Figure $1(\mathrm{e})$ ).

As shown in Figure 2, compared to those in the control group, the fasting serum glucose, fasting serum insulin, and HOMA-IR in the CPAE group were all significantly decreased after 7 weeks' intervention (Figures 2(a), 2(b), and 2(c)). The levels of serum cholesterol, triglyceride, and free fatty acids in the CPAE group were slightly lower than those in control group; only the difference of serum free fatty acids between two groups was statistically significant (Figures 2(d), 2(e), and 2(f)). In addition, the levels of serum GPT and GOT were significantly reduced in the CPAE group, but the level of serum creatinine was not statistically different between two groups (Figures $2(\mathrm{~g}), 2(\mathrm{~h})$, and $2(\mathrm{i})$ ), thus indicating CPAE has no hepatorenal toxicity. Taken together, these data 


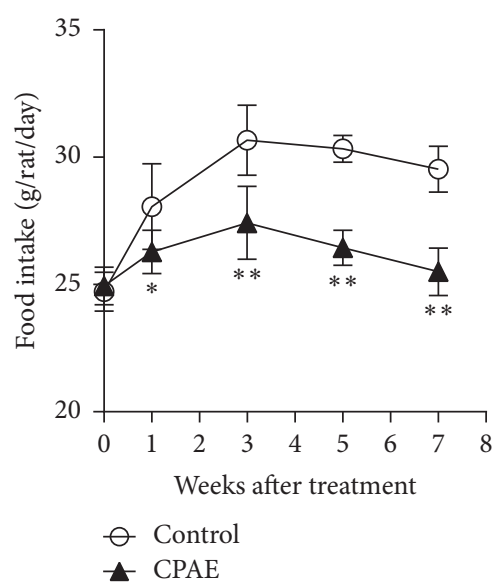

(a)

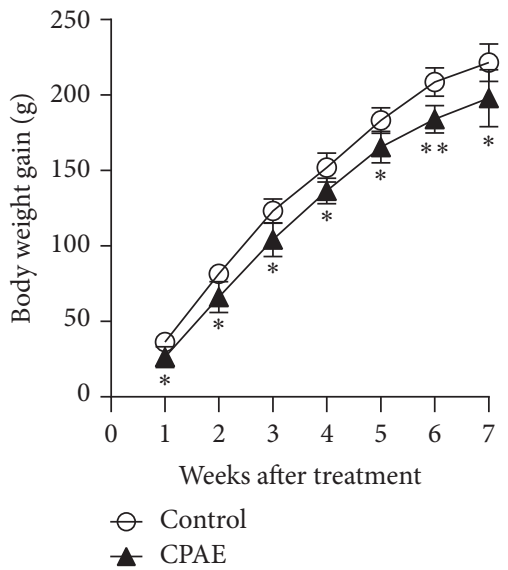

(b)

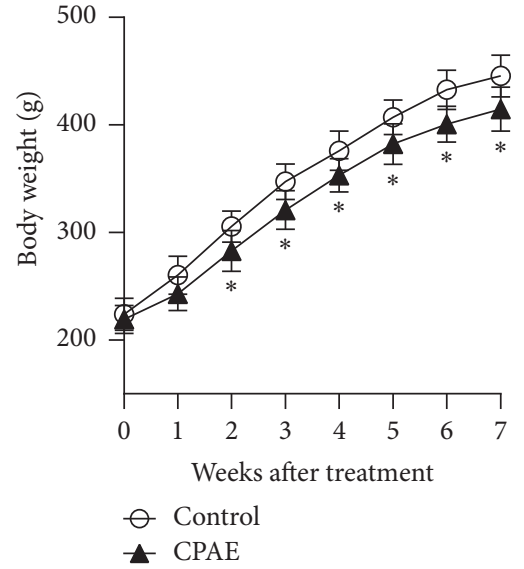

(c)

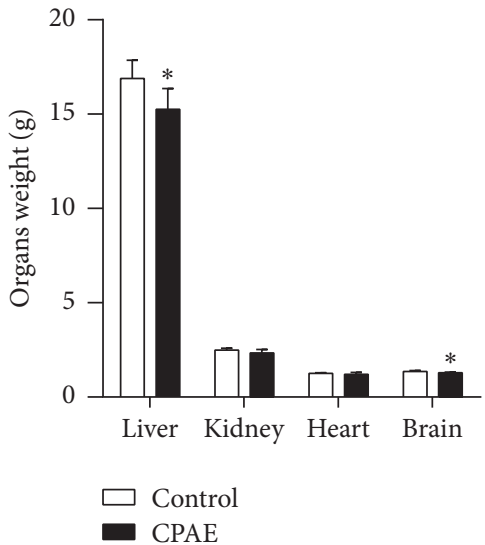

(d)

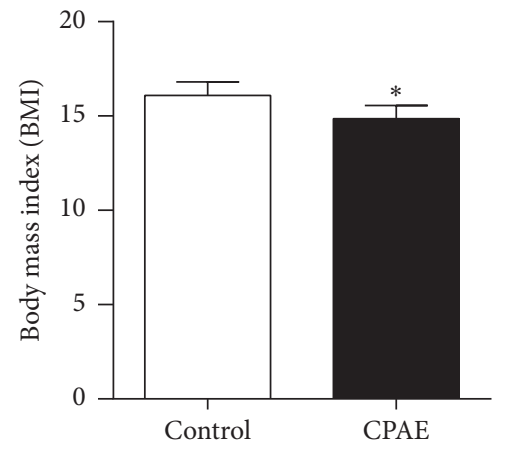

(e)

FIGURE 1: CPAE ameliorates obesity by control of appetite in SHR/cp rats. (a) Body weight, (b) body weight gain, (c) food intake (g/rat/day), (d) organ weight, and (e) body mass index (BMI) of SHR/cp rats treated with $0.5 \mathrm{~g} / \mathrm{kg} \mathrm{CPAE}$ or untreated controls $(n=6)$. Values are expressed as the mean $\pm \mathrm{SD} .{ }^{*} P<0.05$ and ${ }^{* *} P<0.01$ versus control group. $n=6$ per group.

revealed that CPAE can improve obesity, insulin resistance, hyperlipoidemia, and hyperglycemia in SHR/cp rats.

3.2. Antioxidant Potential of CPAE in $S H R / c p$ Rats. The antioxidant potential of CPAE in SHR/cp rats is shown in Figure 3. After treatment with CPAE for 7 weeks, the MDA level in the CPAE group was significantly lower than that in control group (Figure 3(a)). Meanwhile, the levels of SOD and T-GSH in CPAE group were significantly higher than those in the control group (Figures 3(b) and 3(c)). Those results demonstrated the antioxidant potential of CPAE in SHR/cp rats.

3.3. Effects of CPAE on Fat Mass in SHR/cp Rats. After 7 weeks' treatment with CPAE, the fat mass of either the epididymal adipose tissue or the abdominal adipose tissue was significantly reduced, compared to the control group (Figure 4(a)). Furthermore, cell size of abdominal adipocyte in CPAE group was also decreased, compared to the control group (Figure 4(b)). These results could be attributed to the inhibitory effects of CPAE on appetite.
3.4. Effects of CPAE on the Insulin-Signaling Pathway in the Hypothalamus of SHR/cp Rats. Insulin signaling plays an important role in the regulation of gene expression of POMC and NPY. Moreover, InsR and IRS1 are key molecules in the insulin-signaling pathway involved in the activation of downstream targets. To investigate the effects of CPAE on the insulin-signaling pathway in the hypothalamus, activities of several important molecules in this pathway were examined by Western blot analysis. Results showed that phosphorylation levels of InsR and IRS1 (tyr989) in CPAE group were markedly increased, compared to the control group (Figures $5(\mathrm{a})$ and $5(\mathrm{~b})$ ). PI3K, which is activated by upstream p-IRS1 (tyr989), upregulates its downstream target (Akt phosphorylation). PI3K exhibits its effects by translocating to the cell membrane from the cytoplasm $[32,33]$. Our study found that the level of cell membrane PI3Kp85 (m-PI3Kp85) in the CPAE group was significantly higher than in the control group (Figure 5(c)). Thus, the level of p-Akt in CPAE group was higher than that in the control group (Figure 5(d)).

FoxO1 is a transcription factor that is abundant in two neuronal subpopulations characterized by expression of 


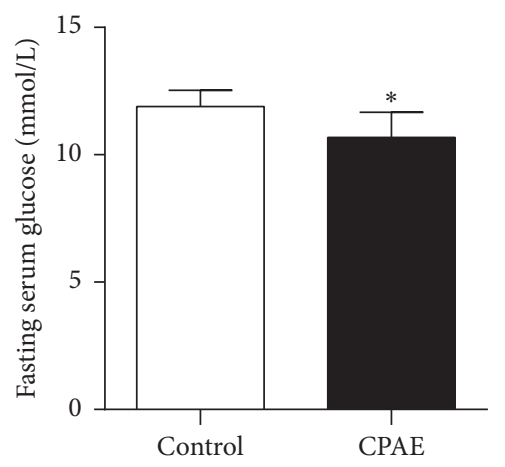

(a)

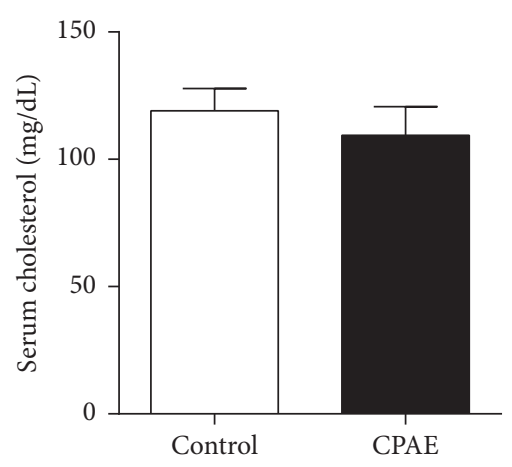

(d)

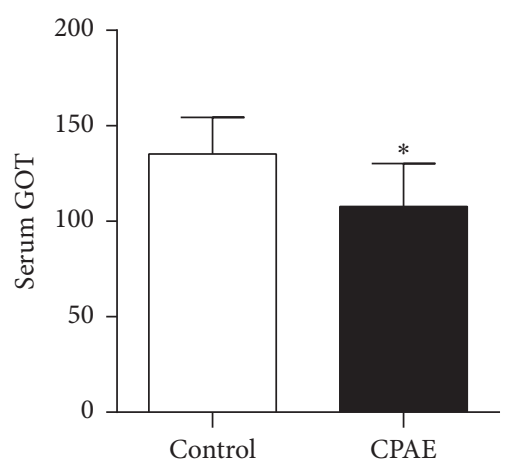

(g)

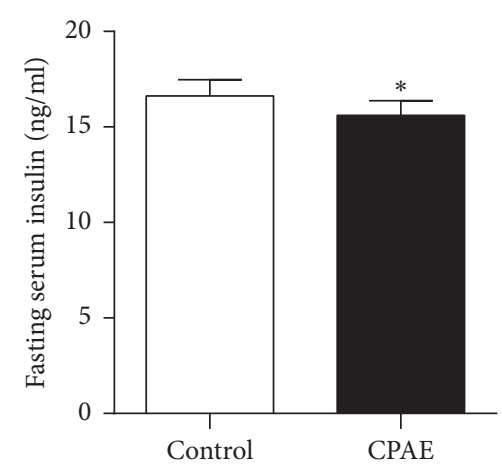

(b)

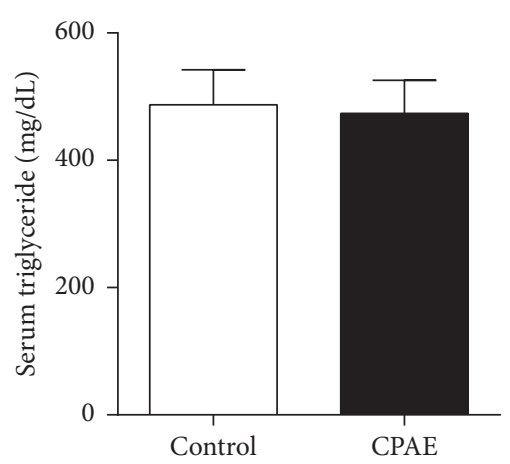

(e)

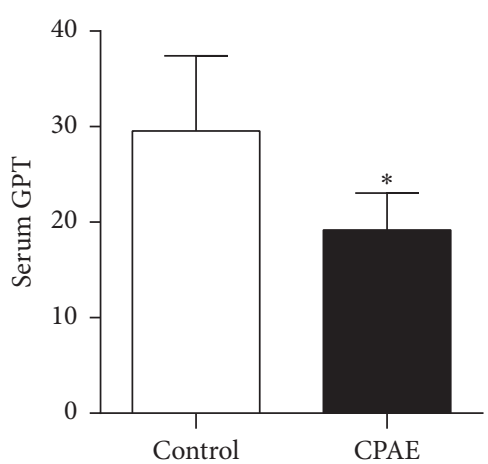

(h)

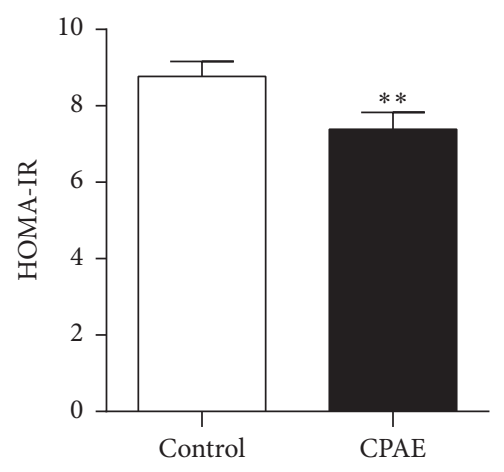

(c)

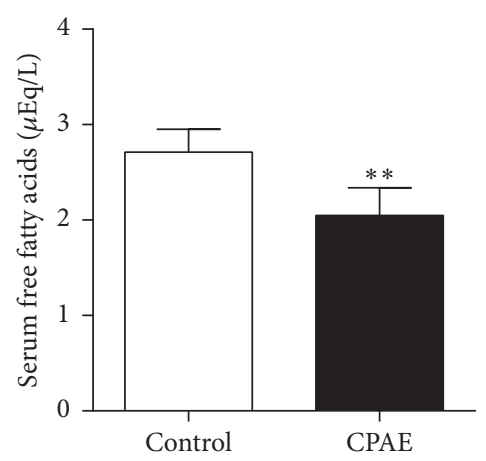

(f)

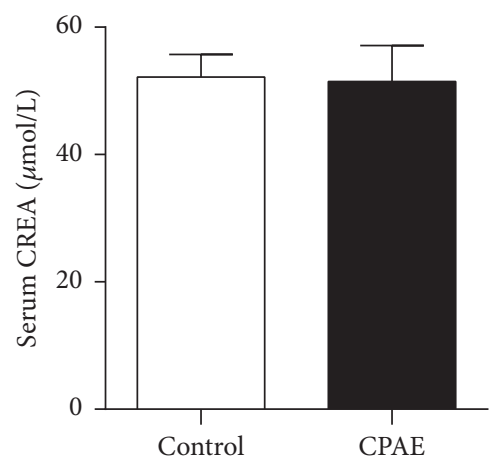

(i)

FIGURE 2: CPAE treatment affects glucose and lipid levels and hepatorenal function in SHR/cp rats. (a) Fasting serum glucose, (b) fasting serum insulin, (c) HOMA-IR, (d) serum cholesterol, (e) serum triglyceride, (f) serum free fatty acids, (g) serum GOT, (h) serum GPT, and (i) serum CREA of SHR/cp rats treated with $0.5 \mathrm{~g} / \mathrm{kg}$ CPAE or untreated controls $(n=6)$ were measured as described in Materials and Methods. Values are expressed as the mean \pm SD. ${ }^{*} P<0.05$ and ${ }^{* *} P<0.01$ versus control group. $n=6$ per group.

POMC or NPY [34]. FoxO1 is inactivated by Akt, whereas activation of FoxO1 promotes food intake by reducing POMC expression and inducing NPY expression. Activation of the insulin-signaling pathway results in the upregulation of p-Akt and sequentially inactivation of FoxO1 due to its phosphorylation by Akt, leading to loss of appetite related to upregulated POMC and downregulated NPY [18].

Our results showed that $\mathrm{p}$-FoxO1 was significantly increased in the CPAE group, compared to the control group. Therefore, POMC expression in the CPAE group was significantly higher than in the control group (Figure 5(f)), although the expression of NPY was decreased (Figure 5(g)). These results demonstrated that CPAE treatment can activate the insulin-signaling pathway in SHR/cp rats.

\section{Discussion}

Obesity is a chronic endocrine metabolic disease associated with hyperglycemia, hyperlipemia, and hypertension [35]. Physically, excessive energy intake due to high food intake is the main cause of obesity. On the other hand, the hypothalamus, a principal center in the brain for regulating appetite, plays an important role in regulation of energy intake [36]. In addition, the insulin-signaling pathway in the hypothalamus is involved in the regulation of appetite [37].

It has been reported that the CPAE can improve insulin resistance by affecting the insulin-signaling pathway in peripheral tissue. However, it is not clear whether CPAE also regulates the insulin-signaling pathway in the hypothalamus. 


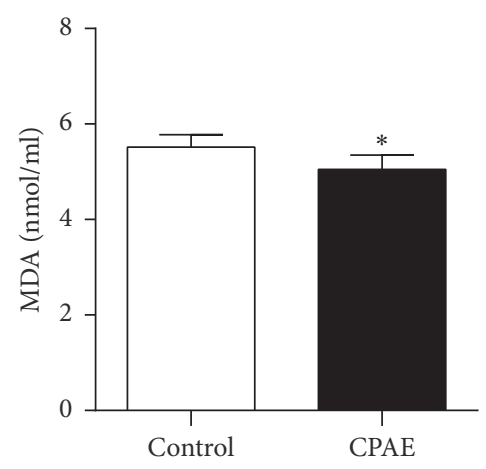

(a)

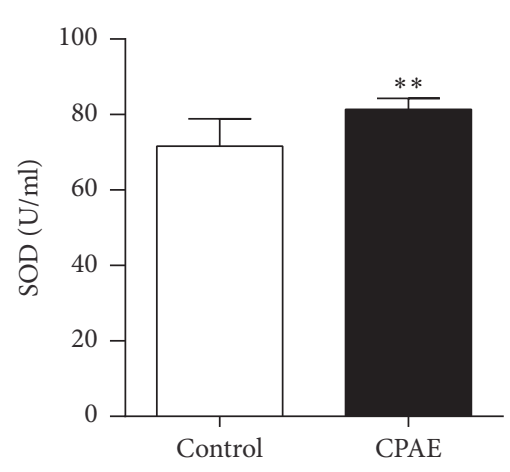

(b)

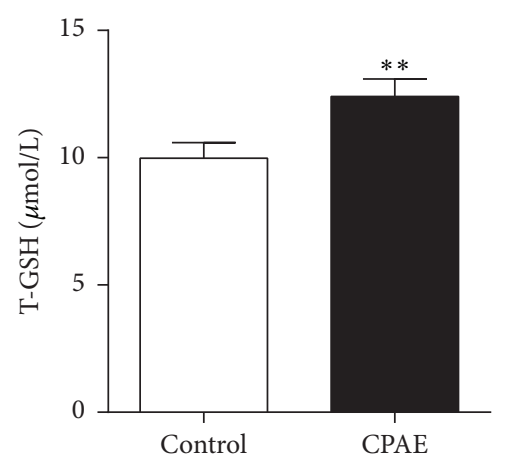

(c)

FIGURE 3: Antioxidant potential of CPAE in SHR/cp rats. (a) MDA, (b) SOD, and (c) the level of T-GSH of SHR/cp rats treated with $0.5 \mathrm{~g} / \mathrm{kg}$ CPAE or untreated controls $(n=6)$ were measured as described in Materials and Methods. Values are expressed as the mean \pm SD. ${ }^{*} P<0.05$ and ${ }^{* *} P<0.01$ versus control group. $n=6$ per group.

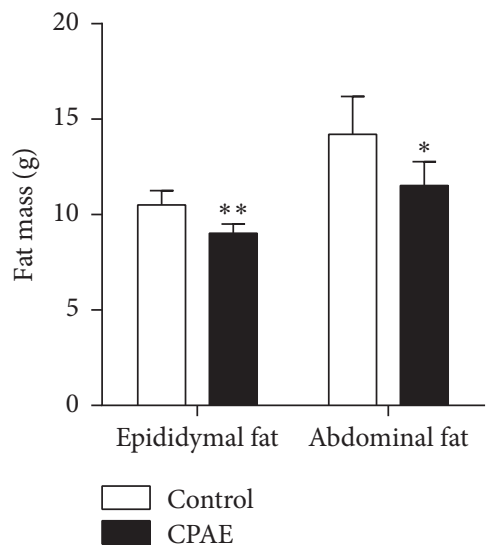

(a)
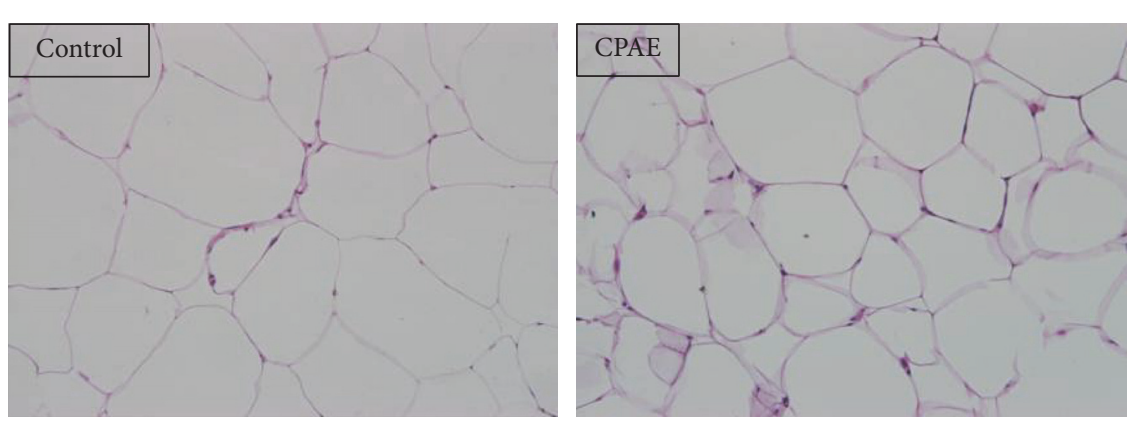

(b)

FIGURE 4: CPAE reduces fat mass in SHR/cp rats. (a) Measurement of fat mass and (b) representative H\&E staining results (magnification $\times 200)$ of the adipose tissue collected from SHR/cp rats which were treated with $0.5 \mathrm{~g} / \mathrm{kg}$ CPAE or untreated controls. Values are expressed as the mean \pm SD. ${ }^{*} P<0.05$ and ${ }^{* *} P<0.01$ versus control group. $n=6$ per group.

Therefore, we investigated the effect of CPAE on the insulin-signaling pathway in hypothalamus, using SHR.CgLeprcp/NDmcr (SHR/cp) rats as a model of obesity and MetS.

In the present study, we demonstrated that treatment with CPAE (0.5 g/kg daily for 7 weeks) can improve obesity, diabetes, hyperglycemia, and hyperlipemia in SHR/cp rats. Our results suggest the effects of CPAE on obesity may be dependent on its ability to decrease food intake. Therefore, a better understanding of the mechanism of antiobesity effects of CPAE is helpful in developing novel antiobesity drugs for treatment of morbid obesity.

The levels of POMC and NPY expression in the hypothalamus are closely associated with the control of food intake. Moreover, the expressions of both POMC and NPY are also controlled by the insulin-signaling pathway in hypothalamus, because NPY neurons are insulin-responsive and appetitestimulating. Indeed, NYP expression can be inhibited by insulin. POMC decreases food intake and reduces body weight in response to insulin stimulation, but the function of POMC is inhibited by neighboring NPY neurons [38, 39].
Previous studies found that insulin receptor (InsR) is widely expressed in the hypothalamus and is involved in the regulation of the insulin-stimulated downstream signaling, while the PI3K/Akt/FoxO1 axis is a key downstream-signaling pathway of InsR and is related to the control of appetite [14].

It is well known that PI3K-dependent Akt activation inhibits FoxO1 activity. Insulin binds to receptors and increases phosphorylation of Akt (one of its downstream targets). Inactivation of FoXO1 by Akt then follows, resulting in upregulation of POMC and downregulation of NPY expression, leading to loss of appetite [19, 40-42]. Therefore, in this study we paid special attention to relationship between CPAE and expression of POMC and NPY. Our data revealed that after 7 weeks' CPAE treatment the level of POMC expression was increased, but NPY expression was reduced. Furthermore, key molecules in the insulin-signaling pathway such as InsR, PI3K, Akt, and FoxOl were activated or inactivated through their phosphorylation. In our study, changes in the phosphorylation of those molecules were found in CPAE-treated SHR/cp rats. In the experimental 


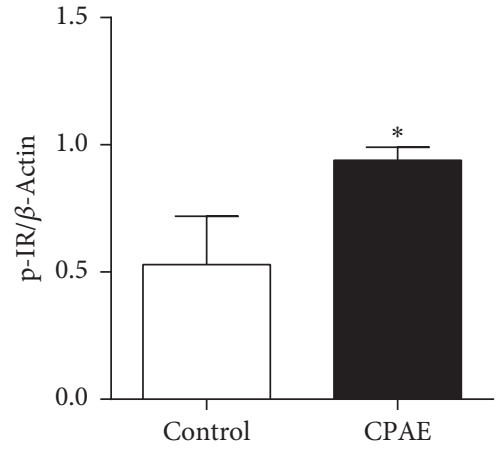

(a)

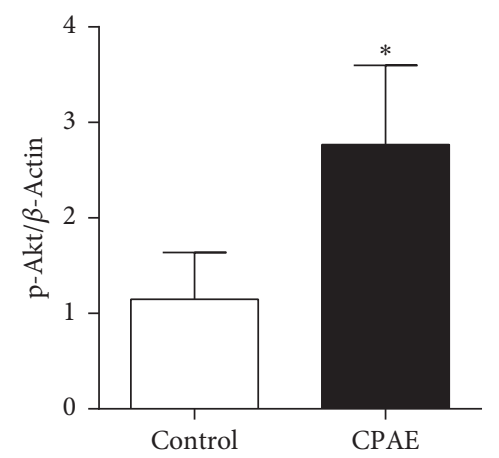

(d)

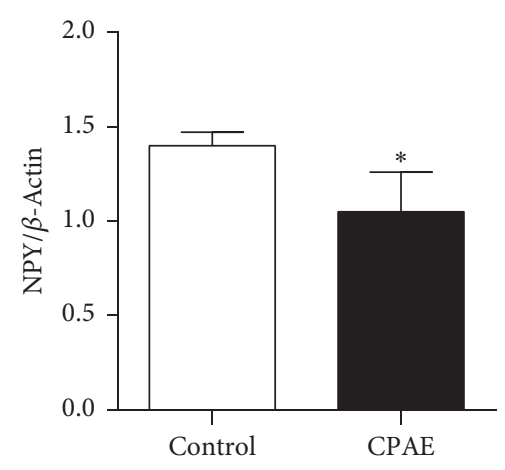

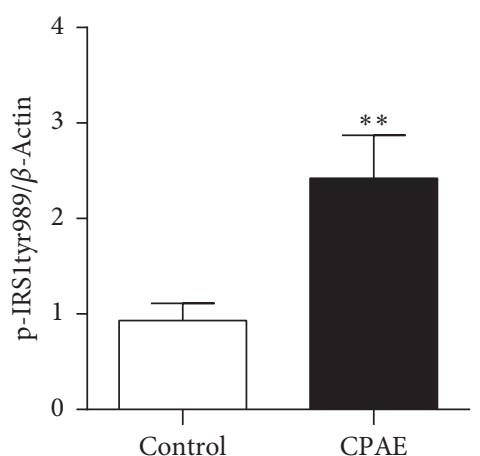

(b)

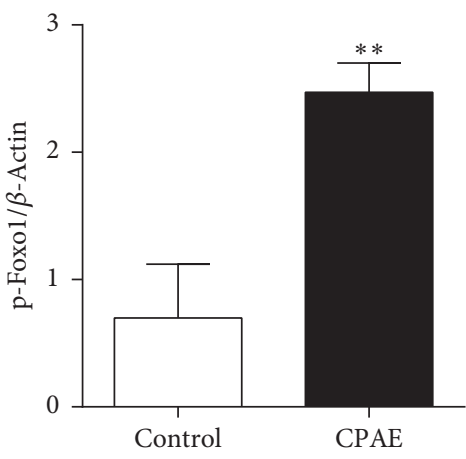

(e)

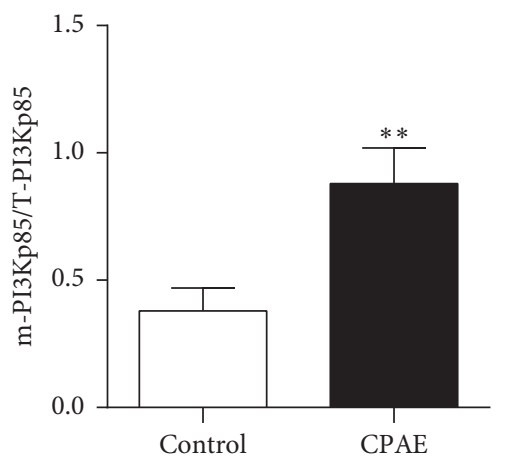

(c)

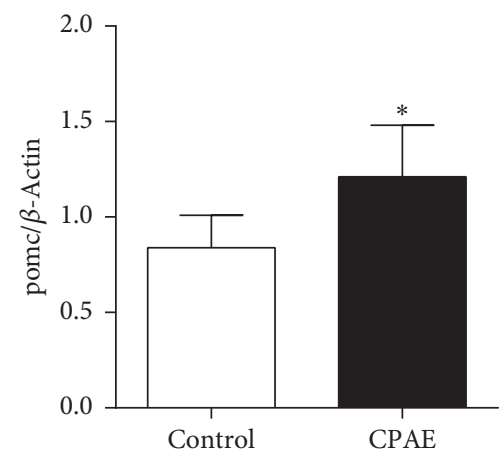

(f)

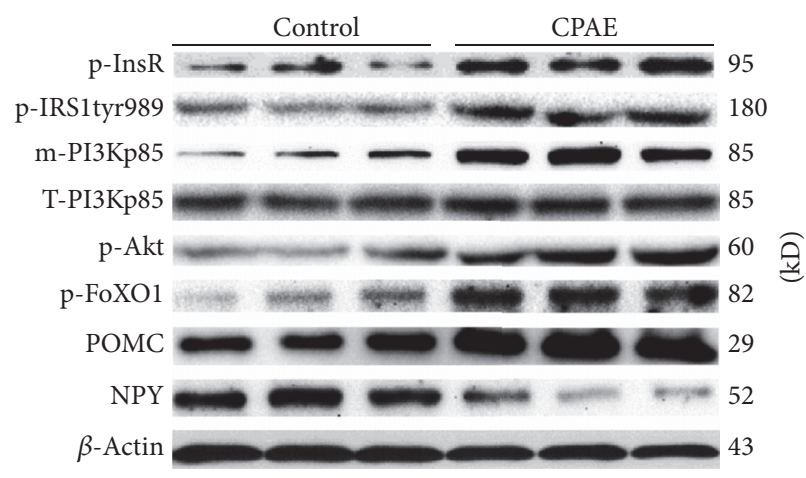

(g)

FIGURE 5: CPAE activates the insulin-signaling pathway in the hypothalamus of SHR/cp rats. (a) The phosphorylation level of InsR, (b) the phosphorylation level of IRS1tyr989, (c) the level of m-PI3Kp85, (d) the phosphorylation level of Akt, (e) the phosphorylation level of FoXO1, (f) the protein expression level of POMC, and (g) NPY of SHR/cp rats treated with $0.5 \mathrm{~g} / \mathrm{kg}$ CPAE or untreated controls. All of the results were determined by Western blot analysis as described in Materials and Methods. Values are expressed as the mean \pm SD. ${ }^{*} P<0.05$ and ${ }^{* *} P<0.01$ versus control group. $n=6$ per group.

rats, those CPAE-mediated changes were correlated with decreased food intake and reduced body weight.

In summary, in this animal study we have demonstrated that CPAE can reduce obesity and insulin resistance and also reduce hyperglycemic, hyperlipidemic, and oxidant stress. Those results are consistent with previously reported studies. We further found that the antiobesity function of CPAE is associated with the suppression of excessive energy intake through a mechanism by activating the insulin-signaling pathway, leading to the inactivation of FoXO1, followed by the upregulation of POMC expression and the downregulation of NPY expression.
Our findings suggest that CPAE may prove to be a promising agent for obesity treatment through regulation of the feeding center of the hypothalamus. In the future, we will further investigate active ingredients and properties of CPAE.

\section{Conflicts of Interest}

The authors declare that they have no conflicts of interest.

\section{Acknowledgments}

The International Scientific Collaborative Project (2010DFB33260) and the Innovative team of Beijing 
University of Chinese Medicine (2011-CXTD-19) funded this research. International Joint Research Center of Prevention and Treatment of Diabetes with Traditional Chinese Medicine of Ministry of Science and Technology of China; Key Laboratory of Health Cultivation of the Ministry of Education; Key Laboratory of Health Cultivation of Beijing (BZ0259); Beijing International Scientific and Technological Cooperation Base for the Prevention and Treatment of Diabetes with Traditional Chinese Medicine are acknowledged.

\section{References}

[1] K. F. Adams, A. Schatzkin, T. B. Harris et al., "Overweight, obesity, and mortality in a large prospective cohort of persons 50 to 71 years old," The New England Journal of Medicine, vol. 355, no. 8, pp. 763-778, 2006.

[2] M. Ng, T. Fleming, M. Robinson, and et al, "Global, regional, and national prevalence of overweight and obesity in children and adults during 1980-2013: a systematic analysis for the Global Burden of Disease Study 2013," The Lancet, vol. 384, no. 9945, pp. 766-781, 2014.

[3] J. O. Hill, H. R. Wyatt, and J. C. Peters, "Energy balance and obesity," Circulation, vol. 126, no. 1, pp. 126-132, 2012.

[4] M. K. Olsen, H. Johannessen, N. Cassie et al., "Steady-state energy balance in animal models of obesity and weight loss," Scandinavian Journal of Gastroenterology, vol. 52, no. 4, pp. 442449, 2016.

[5] A. P. Coll, I. S. Farooqi, B. G. Challis, G. S. H. Yeo, and S. O'Rahilly, "Proopiomelanocortin and energy balance: insights from human and murine genetics," Journal of Clinical Endocrinology and Metabolism, vol. 89, no. 6, pp. 2557-2562, 2004.

[6] H.-R. Berthoud, "Metabolic and hedonic drives in the neural control of appetite: who is the boss?" Current Opinion in Neurobiology, vol. 21, no. 6, pp. 888-896, 2011.

[7] D. Nuzzaci, A. Laderrière, A. Lemoine et al., "Plasticity of the melanocortin system: determinants and possible consequences on food intake," Frontiers in Endocrinology, vol. 6, 2015.

[8] V. Y. Susanti, T. Sasaki, H. Yokota-Hashimoto et al., "Sirt1 rescues the obesity induced by insulin-resistant constitutivelynuclear FoxO1 in POMC neurons of male mice," Obesity, vol. 22, no. 10, pp. 2115-2119, 2014.

[9] G. A. Bewick, J. V. Gardiner, W. S. Dhillo et al., "Post-embryonic ablation of AgRP neurons in mice leads to a lean, hypophagic phenotype," FASEB Journal, vol. 19, no. 12, pp. 1680-1682, 2005.

[10] R. Stark, A. Reichenbach, and Z. B. Andrews, "Hypothalamic carnitine metabolism integrates nutrient and hormonal feedback to regulate energy homeostasis," Molecular and Cellular Endocrinology, vol. 418, pp. 9-16, 2015.

[11] J. Havrankova, J. Roth, and M. Brownstein, "Insulin receptors are widely distributed in the central nervous system of the rat," Nature, vol. 272, no. 5656, pp. 827-829, 1978.

[12] A. C. Hausen, J. Ruud, H. Jiang et al., "Insulin-dependent activation of mch neurons impairs locomotor activity and insulin sensitivity in obesity," Cell Reports, vol. 17, no. 10, pp. 2512-2521, 2016.

[13] T. Kitamura, "Obesity and the central regulation of energy metabolism," Nihon Rinsho, vol. 71, no. 2, pp. 217-223, 2013.

[14] K. D. Niswender, C. D. Morrison, D. J. Clegg et al., "Insulin activation of phosphatidylinositol 3-kinase in the hypothalamic arcuate nucleus: A key mediator of insulin-induced anorexia," Diabetes, vol. 52, no. 2, pp. 227-231, 2003.

[15] M. F. M. Hoekman, F. M. J. Jacobs, M. P. Smidt, and J. P. H. Burbach, "Spatial and temporal expression of FoxO transcription factors in the developing and adult murine brain," Gene Expression Patterns, vol. 6, no. 2, pp. 134-140, 2006.

[16] Y. Jeon, S. Aja, G. V. Ronnett, and E.-K. Kim, "D-chiro-inositol glycan reduces food intake by regulating hypothalamic neuropeptide expression via AKT-FoxO1 pathway," Biochemical and Biophysical Research Communications, vol. 470, no. 4, pp. 818-823, 2016.

[17] R. Chruvattil, S. Banerjee, S. Nath et al., "Dexamethasone alters the appetite regulation via induction of hypothalamic insulin resistance in rat brain," Molecular Neurobiology, pp. 1-14.

[18] L. Plum, H. V. Lin, K. S. Aizawa, Y. Liu, and D. Accili, "InsR/ FoxO1 signaling curtails hypothalamic POMC neuron number," PLoS ONE, vol. 7, no. 2, Article ID e31487, 2012.

[19] K. Iskandar, Y. Cao, Y. Hayashi et al., "PDK-1/FoxO1 pathway in POMC neurons regulates Pomc expression and food intake," American Journal of Physiology - Endocrinology and Metabolism, vol. 298, no. 4, pp. E787-E798, 2010.

[20] H.-J. Kim, M. Kobayashi, T. Sasaki et al., "Overexpression of FoxO1 in the hypothalamus and pancreas causes obesity and glucose intolerance," Endocrinology, vol. 153, no. 2, pp. 659-671, 2012.

[21] S. A. He, Chinese Rare Plants Shanghai, vol. 19, Shanghai Science and Technology Press, Shanghai, China, 1988.

[22] Plant Index Editorial Committee of the Chinese Academy of Science, The Plant Index of China, vol. 23, Scientific Press, Beijing, China, 1979.

[23] Q. Wang, C. Jiang, S. Fang et al., "Antihyperglycemic, antihyperlipidemic and antioxidant effects of ethanol and aqueous extracts of Cyclocarya paliurus leaves in type 2 diabetic rats," Journal of Ethnopharmacology, vol. 150, no. 3, pp. 1119-1127, 2013.

[24] K. Wang and Y. Cao, "Research progress in the chemical constituents and pharmacologic activities of Cyclocaryapaliurus (Batal.) Iljinshaja," Heilongjiang Medical Journal, vol. 31, pp. 577-579, 2007.

[25] C. Jiang, N. Yao, Q. Wang et al., "Cyclocarya paliurus extract modulates adipokine expression and improves insulin sensitivity by inhibition of inflammation in mice," Journal of Ethnopharmacology, vol. 153, no. 2, pp. 344-351, 2014.

[26] Y. Ma, C. Jiang, N. Yao et al., "Antihyperlipidemic effect of Cyclocarya paliurus (Batal.) Iljinskaja extract and inhibition of apolipoprotein B48 overproduction in hyperlipidemic mice," Journal of Ethnopharmacology, vol. 166, pp. 286-296, 2015.

[27] K. N. Zhu, C. H. Jiang, Y. S. Tian et al., "Two triterpeniods from Cyclocarya paliurus (Batal) Iljinsk (Juglandaceae) promote glucose uptake in 3T3-L1 adipocytes: the relationship to AMPK activation," Phytomedicine, vol. 22, no. 9, pp. 837-846, 2015.

[28] K. Kawai, T. Sakairi, S. Harada et al., "Diet modification and its influence on metabolic and related pathological alterations in the SHR/NDmcr-cp rat, an animal model of the metabolic syndrome," Experimental and Toxicologic Pathology, vol. 64, no. 4, pp. 333-338, 2012.

[29] J.-H. Xie, M.-Y. Xie, S.-P. Nie, M.-Y. Shen, Y.-X. Wang, and C. Li, "Isolation, chemical composition and antioxidant activities of a water-soluble polysaccharide from Cyclocarya paliurus (Batal.) Iljinskaja," Food Chemistry, vol. 119, no. 4, pp. 1626-1632, 2010.

[30] J.-H. Xie, M.-Y. Xie, M.-Y. Shen, S.-P. Nie, C. Li, and Y.$\mathrm{X}$. Wang, "Optimisation of microwave-assisted extraction of 
polysaccharides from Cyclocarya paliurus (Batal.) Iljinskaja using response surface methodology," Journal of the Science of Food and Agriculture, vol. 90, no. 8, pp. 1353-1360, 2010.

[31] D. R. Matthews, J. P. Hosker, A. S. Rudenski, B. A. Naylor, D. F. Treacher, and R. C. Turner, "Homeostasis model assessment: insulin resistance and $\beta$-cell function from fasting plasma glucose and insulin concentrations in man," Diabetologia, vol. 28, no. 7, pp. 412-419, 1985.

[32] L. C. Cantley, "The phosphoinositide 3-kinase pathway," Science, vol. 296, no. 5573, pp. 1655-1657, 2002.

[33] M. Sata and R. Nagai, "Phosphatidylinositol 3-kinase: a key regulator of vascular tone?" Circulation Research, vol. 91, no. 4, pp. 273-275, 2002.

[34] M.-S. Kim, Y. K. Pak, P.-G. Jang et al., "Role of hypothalamic Foxol in the regulation of food intake and energy homeostasis," Nature Neuroscience, vol. 9, no. 7, pp. 901-906, 2006.

[35] G. R. Hajer, T. W. Van Haeften, and F. L. J. Visseren, "Adipose tissue dysfunction in obesity, diabetes, and vascular diseases," European Heart Journal, vol. 29, no. 24, pp. 2959-2971, 2008.

[36] G. J. Morton, D. E. Cummings, D. G. Baskin, G. S. Barsh, and M. W. Schwartz, "Central nervous system control of food intake and body weight," Nature, vol. 443, no. 7109, pp. 289-295, 2006.

[37] M. Thon, T. Hosoi, and K. Ozawa, "Possible integrative actions of leptin and insulin signaling in the hypothalamus targeting energy homeostasis," Frontiers in Endocrinology, vol. 7, 2016.

[38] J.-W. Sohn, "Network of hypothalamic neurons that control appetite," BMB Reports, vol. 48, no. 4, pp. 229-233, 2015.

[39] L. Shanshan, Z. Cuizhen, P. Gang et al., "Effects of starvation on the expression of feeding related neuropeptides in the larval zebrafish hypothalamus," Yi Chuan, vol. 38, no. 9, pp. 821-830, 2016.

[40] H. V. Lin, L. Plum, H. Ono et al., "Divergent regulation of energy expenditure and hepatic glucose production by insulin receptor in agouti-related protein and POMC neurons," Diabetes, vol. 59, no. 2, pp. 337-346, 2010.

[41] X. Guan, "The CNS glucagon-like peptide-2 receptor in the control of energy balance and glucose homeostasis," American Journal of Physiology - Regulatory Integrative and Comparative Physiology, vol. 307, no. 6, pp. R585-R596, 2014.

[42] E. Burgos-Ramos, Á. González-Rodríguez, S. Canelles et al., "Differential insulin receptor substrate-1 (IRS1)-related modulation of neuropeptide $y$ and proopiomelanocortin expression in nondiabetic and diabetic IRS2 -/- mice," Endocrinology, vol. 153, no. 3, pp. 1129-1140, 2012. 


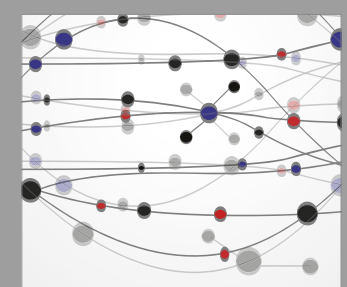

The Scientific World Journal
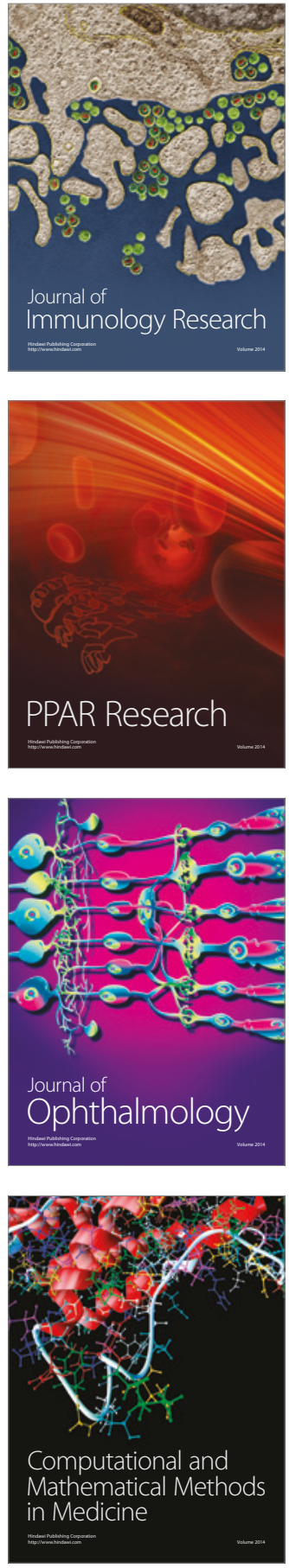

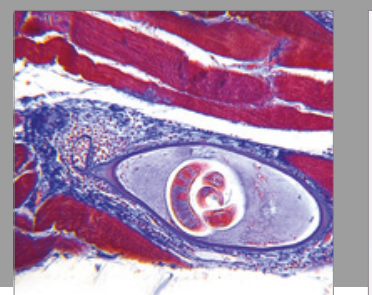

Gastroenterology Research and Practice
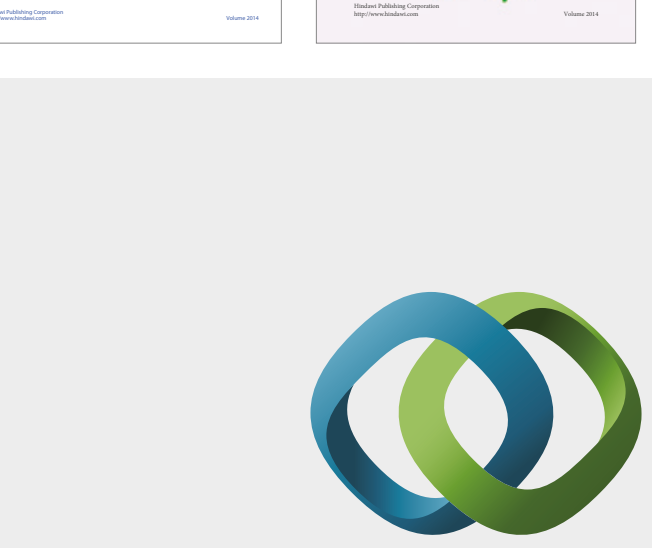

\section{Hindawi}

Submit your manuscripts at

https://www.hindawi.com
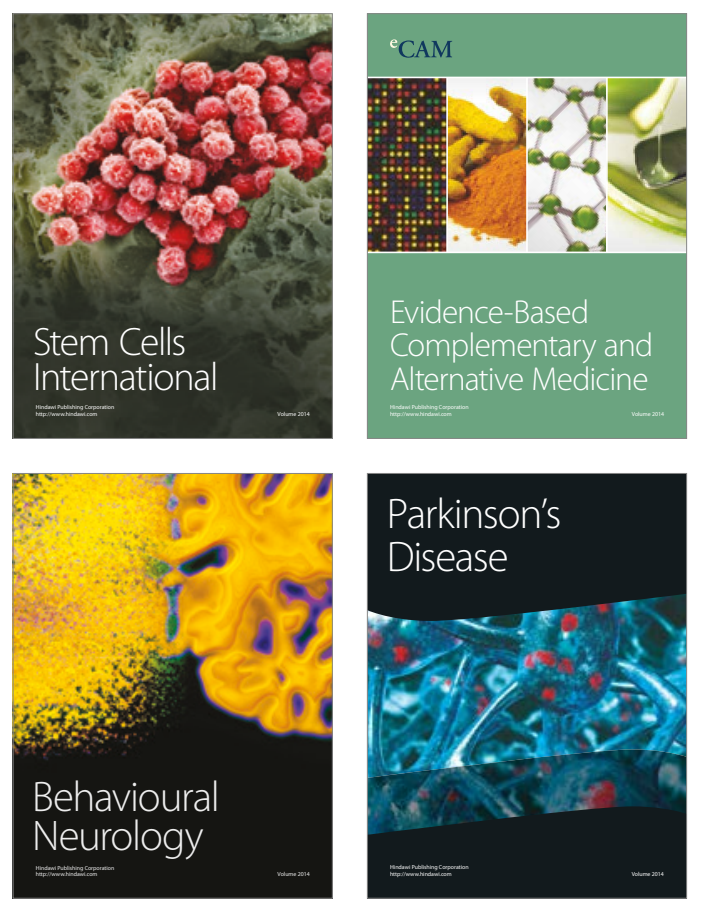
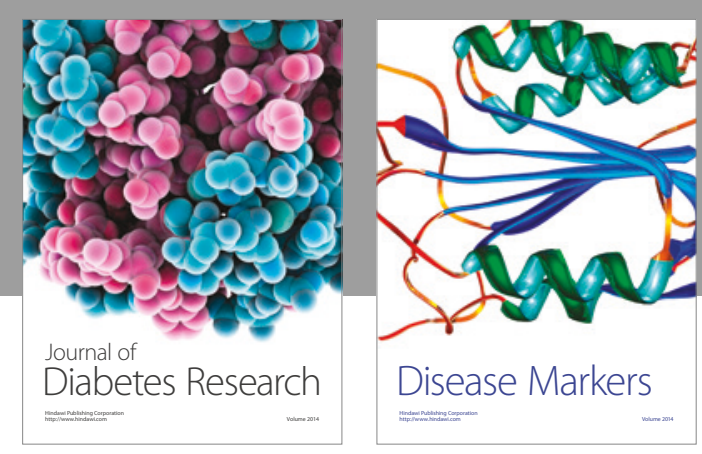

Disease Markers
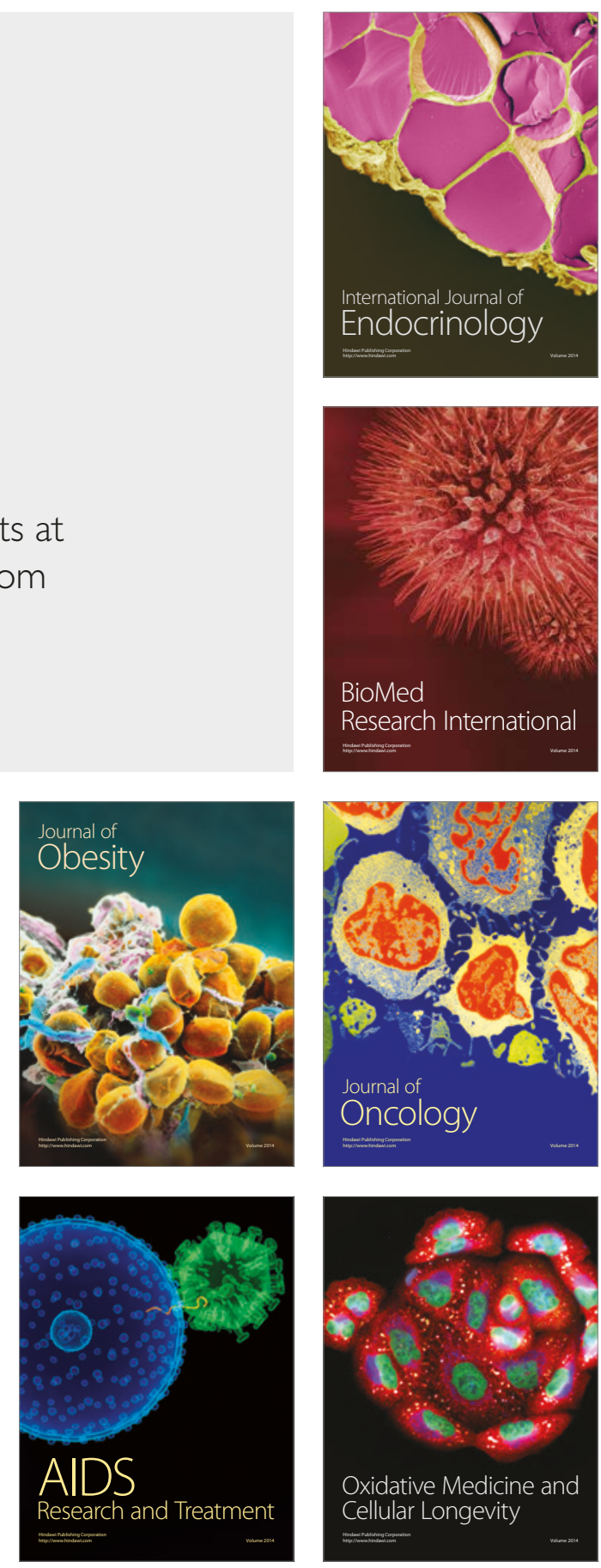